\title{
Realidade Aumentada com em um Mapa Tátil Sonoro para Deficientes Visuais: um sistema de interação.
}

\author{
Roberto Sussumu Wataya ${ }^{1}$, João V. V. d'Abreu ${ }^{2}$, Claudio Kirner ${ }^{3}$, Nubia Bernardi ${ }^{4}$ \\ ${ }^{1}$ Departamento de Computação - Centro Universitário Adventista de São Paulo- \\ UNASP. Estrada de Itapecerica, 5859- Jd IAE- São Paulo, SP, Brazil. CEP 05859-001 e \\ NIED/UNICAMP. Cidade Universitária Zeferino Vaz, Rua 06 de agosto, 50. Campinas, \\ SP, Brazil. CEP 13083-873 \\ ${ }^{2}$ NIED/UNICAMP. Cidade Universitária Zeferino Vaz, Rua 06 de agosto, 50. \\ Campinas, SP, Brazil. CEP 13083-873. \\ ${ }^{3}$ Universidade Federal de Itajubá- UNIFEI. Av. BPS, 1303- Bairro Pinheirinho, Itajubá- \\ MG, Brasil, CEP 37500903. \\ ${ }^{4}$ Faculdade de Engenharia Civil Arquitetura e Urbanismo- FEC/UNICAMP- Cidade \\ Universitária Zeferino Vaz, Av. Albert Einstein, 951, SP, Brazil, CEP 13083-852. \\ \{roberto.sussumu@unasp.edu.br, jvilhete@unicamp.br, ckirner@unifei.edu.br, nubiab@unicamp.br \}
}

Abstract. This study describes the creation and implementation of 3D-System Interaction 3D SI3D-using two systems, the first is the FLARAS-Flash Augmented Reality Authoring System, which runs directly from a web browser using Adobe Flash Player so online or local. The second is the Tactile Sound Map-MTS, of a particular route called Route Accessible at Unicamp in Barão São Geraldo, Campinas, SP., which is an embedded system with 3 circuits: plate reader sensors based on the use of the micro-controller (PIC microchip ), Arduino controller board and circuit implementation of sounds (voice chip). The system SI3D associated with MTS will allow users of the route to be performed, additional information such as: sound, 3D imagery, textual and libras.

Resumo. Este estudo descreve a criação e implementação do Sistema de Interação 3DSI3D- utilizando dois sistemas, o primeiro é o FLARAS- Flash Augmented Reality Authoring System- que é executada diretamente do navegador de internet por meio do Adobe Flash Player de forma online ou local. O segundo é o Mapa Tátil Sonoro de um determinado percurso denominado Rota Acessível na Unicamp em Barão São Geraldo, Campinas, SP., que é um sistema embarcado com 3 circuitos distintos: placa leitor de sensores fundamentada no uso do micro-controlador PIC (microship), placa controladora Arduino e o circuito de execução de sons (chip voice). O sistema SI3D associado ao MTS possibilitará aos usuários do percurso a ser realizado, informações adicionais como: sonora, imagética 3D, textual e libras.

\section{INTRODUÇÃ̃O}

O professor Claudio Kirner, um dos precursores da Realidade Aumentada- RA, tem se destacado com as pesquisas e aplicações educacionais para pessoas com necessidades educativas especiais (KIRNER, 2004; KIRNER, 2007; KIRNER \& KIRNER, 2007; KIRNER \& TORI, 2006; SANTIN \& KIRNER, 2005). Percebe-se assim, a preocupação com iniciativas de educação inclusiva, que podem transformar o ensino em uma prática real e eficaz para pessoas com deficiência visual, tendo como base os aspectos sonoros e táteis, que podem ser simulados em um sistema de RA. Assim, ambientes de simulação e interação 3D baseado em RA, permitem o estudo e análise de forma realista de casos pontuais, novas modalidades e metodologias (WATAYA, 2010). 
Para este estudo, foram empregadas duas ferramentas tais como: o Uso da Realidade Aumentada com o FLARAS e o Mapa tátil e Sonoro. A primeira é uma ferramenta de autoria de realidade aumentada, nomeada como FLARAS ${ }^{1}$ - Flash Augmented Reality Authoring System, e a segunda ferramenta é o sistema de controle do Mapa Tátil Sonoro-MTS ${ }^{2}$,

Com base nos dois sistemas descritos, esse trabalho tem como objetivo desenvolver um Sistema de Interação 3D, utilizando o Flaras com o Mapa Tátil Sonoro que dê aos deficientes visuais e surdos, mais informações adicionais como: sonora, imagética 3D, textual e libras.

O artigo apresenta, na seção 2, A estrutura do SI3D-DV e suas características. Na seção 3, O Mapa Tátil sonoro, na seção 4, Estruturação do SI3D-DV com FLARAS, na seção 5, apresenta A Utilização do SI3D-DV no mapa tátil e, finalmente na seção 6, destaca Considerações finais do estudo.

\section{Estrutura do Sistema Interação 3D para Deficientes Visuais - SI3D-DV 2.1. Descrição do FLARAS}

O FLARAS é uma ferramenta de autoria derivada de realidade aumentada e foi desenvolvida por Raryel C. Souza e Hipólito Douglas F. Moreira, sob a orientação de Claudio Kirner. Além disso, o FLARAS utiliza a mesma estrutura do SACRA ${ }^{3}$ de cadastramento de pontos e cenas, por isso, ele representa uma evolução e em especial, é destacado a facilidade de seu uso, pois sua interface gráfica é amigável e também as aplicações desenvolvidas são executadas na modalidade online como offline (sem internet) em qualquer sistema operacional no qual exista o Adobe Flash Player. Este último representa uma grande vantagem, o da computação em nuvem (cloud computing). Entre as melhorias, podemos destacar a possibilidade de adicionar vídeos nas aplicações, bem como objetos virtuais do armazém 3D do Google e áudio em MP3 (SOUZA, MOREIRA, KIRNER, 2012).

\subsection{Modos do marcador de interação}

O marcador de interação pode executar diferentes ações em um ponto, dependendo do "modo de operação" selecionado. A alternância entre os modos é feito através do teclado. Os modos de operação do marcador de interação são mostrados na Figura 3.

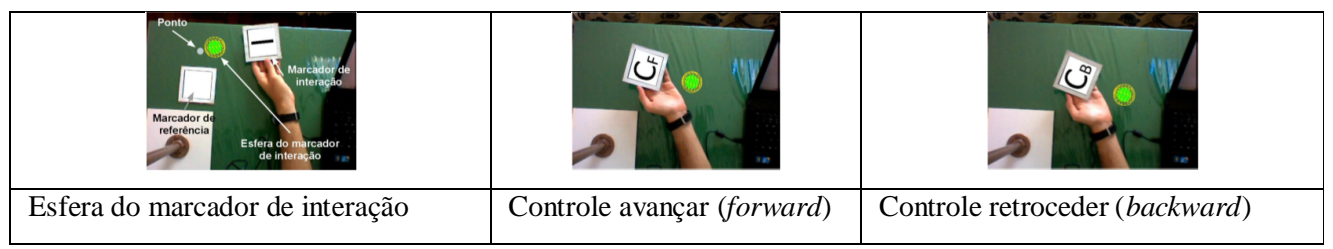

Figura 3 - Marcador de interação.

Fonte: SOUZA, MOREIRA e KIRNER, 2012.

"Fazer uma ação de controle em um ponto", isto é, usar o marcador de interação, modo de operação controle, em um ponto, permite avançar ou retroceder na lista de cenas associadas ao ponto. Essa ação só funciona em pontos ativados. Para retroceder o marcador tem de estar no modo "controle backward", e para avançar, ele deve estar no modo "controle forward". Caso se faça uma ação de controle avançando na última cena

\footnotetext{
${ }^{1}$ FLARAS- será explicado no item 2.1

${ }^{2}$ MTS- será explicado no item 3

${ }^{3}$ SACRA- Sistema de Autoria Colaborativa com Realidade Aumentada, software utilizada com a colaboração de vários autores, com participações locais e remotamente.
} 
da lista, automaticamente se retornará para a primeira cena da lista. O mesmo é válido para uma ação de controle retrocedendo na primeira cena da lista (SOUZA, MOREIRA e KIRNER, 2012).

\section{Mapa Tátil e sonoro}

Do ponto de vista tecnológico, o mapa tátil sonoro constitui-se de "um ambiente de processamento de sinais elétricos baseado em sensores que possuem uma interface de hardware onde estão conectados e, de um processador que executa a informação e a 'pronúncia' de uma determinada frase quando um dos sensores é pressionado". A cada botão sensor está associado um objeto representado no mapa.

Na superfície superior do mapa tátil sonoro há uma "implantação" que reproduz o que seria a vista aérea do campus da Unicamp, na qual são representadas algumas edificações. Por meio de relevos e texturas distintas, "prédios-chave" e caminhos são destacados. Ao lado destes há botões que, ao serem acionados, liberam uma informação sonora (voz previamente gravada) do tipo "Você está no Ciclo Básico" (d'ABREU, 2011), essa descrição é confirmada na Figura5.

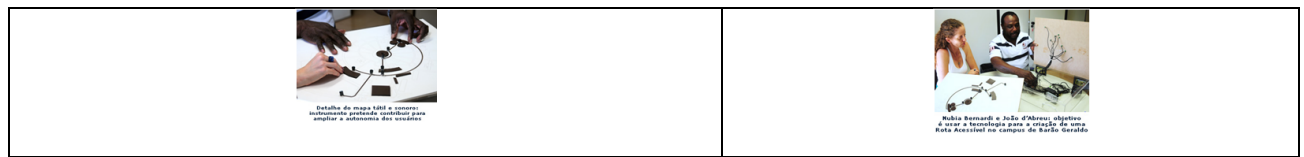

Figura 5 - Mapa Tátil sonoro ${ }^{4}$

Fonte: Copyright (C) 2002-2008 Nied - Núcleo de Informática Aplicada à Educação.

\section{Estruturação do SI3D-DV com FLARAS}

O Sistema de Interação 3D para Deficientes Visuais- SI3D-DV, está sendo implementado, com o objetivo de viabilizar um sistema interativo-participativo, permitindo uma ampla interação entre o cego ou outro usuário, objeto real, objeto virtual e o sistema mapa de uso tátil e sonoro, da rota acessível de uma parte do campus da Universidade Estadual de Campinas. Também, deve proporcionar situações de conhecimento e aprendizagem do espaço geográfico e permitir interações com novos locais físicos, facilitando aos DV o domínio e a apreensão de novos conceitos e localização dos espaços até então desconhecidos.

O uso da realidade aumentada com o FLARAS associado ao Mapa Tátil e Sonoro na construção do SI3D-DV, são baseados nos atributos como: necessidade de comunicação, conhecimento / reconhecimento de um dado espaço e capacidade de uso da "memória visual", de pessoas com deficiência visual, atributos estes que são capturados e construídos por meio do tato, das informações conceituais e sonoras dos objetos quando manipulado por essas pessoas (WATAYA, 2010).

\section{Utilização do SI3D- DV no Mapa Tátil e Sonoro- MTS}

Para a utilização do SI3D - DV, criou se um cenário virtual a partir do Mapa Tátil Sonoro com todos os objetos que o compõe. Para melhor compreensão, vamos descrever como se processa tal sistema: Na figura 6, temos o cenário da "Rota Acessível", o SI3D-DV e um dos pontos importantes da rota. Quando o ponto é tocado, há uma colisão dos pontos virtuais do "prédio" com o da placa de identificação do objeto, que resulta no surgimento do referido prédio virtual aliada aos recursos sonoro, imagético e libras.

\footnotetext{
${ }^{4}$ Figura 5 - disponível no website: $\underline{\text { http://pan.nied.unicamp.br/novidades/novidade_completa.php?id=48 }}$ acesso em 22/mai/2013.
} 


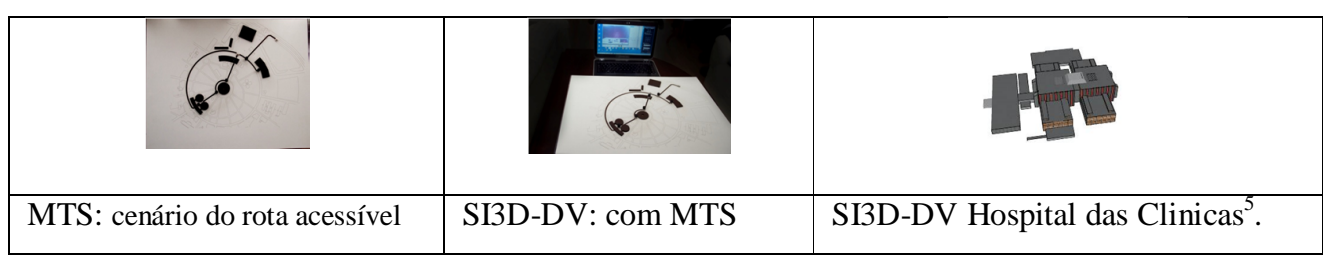

Figura 6 - O SI3D-DV em ação.

Fonte: Roberto Wataya

Dessa forma, foram observados que sua utilização ainda que parcial, foi de fácil interação e a seguir no próximo ítem, faremos as considerações finais.

\section{Considerações Finais}

O Sistema de Interação 3D para Deficientes Visuais, permite a interação com os usuários, proporcionando condições de assimilar os objetos que compõe o lay-out da rota acessível e promovendo mais autonomia no seu deslocamento na rota acessível do campus. O SI3D-DV também é adequado para pessoas com deficiências visuais e surdos, pois conjuga os sentidos do tato, de audição e libras para a sua compreensão do espaço representado. Aos usuários sem deficiência, o sistema aciona todos os recursos multi-sensoriais aplicados na orientação espacial do campus como imagens, som, tato, texto e libras.

Por fim, este trabalho tem procurado estudar as técnicas e as necessidades que envolvem uma pessoa com deficiência visual e surdos, para o desenvolvimento do SI3D-DV. Entretanto, o desenvolvimento das pesquisas tem denotado que a ferramenta em construção não só trará, contribuição significativa para a inclusão da pessoa com deficiência visual, mas que também poderá ser para outras pessoas, inclusive sem deficiência.

\section{Referências Bibliográficas}

d'ABREU, J.V.V. Orientação Espacial no Campus da Unicamp: Desenvolvimento de um mapa de uso tátil e sonoro como ferramenta de auxílio ao percurso do usuário. 2011. Disponível no website: http//www.unicamp.br/ acesso em 29/junho/2012.

KIRNER, C. Mãos Colaborativas em Ambientes de Realidade Misturada. Anais do $1^{\circ}$ Workshop de Realidade Aumentada. Piracicaba, SP, 2004, p. 1-4.

KIRNER, C.; KIRNER, T.C. Virtual Reality and Augmented Reality Applied to Simulation Visualization. In: El Sheikh, A.A.R.; Al Ajeeli, A.; Abu-Taieh, E.M.O..(Org.). Simulation and Modeling: Current Technologies and Applications. 1 ed. Hershey-NY: IGI Publishing, 2007, v.1, p.391-419.

KIRNER, C.; TORI, R. Fundamentos de Realidade Aumentada. In: Claudio Kirner; Romero Tori; Robson Siscoutto (Ed.) Fundamentos e Tecnologia de Realidade Virtual e Aumentada. Pré Simpósio SVR 2006, SBC, Belém/PA., 2006, p.22-38.

SANTIN, R.; KIRNER, C. Detecção em Tempo Real de Objetos em Vídeo Usando Realidade Aumentada. In: Anais do WRA' 2005- II Workshop de Realidade Aumentada, 2005, Piracicaba, SP. V1, p.25-28.

SOUZA, R.C.; MOREIRA, H. D. F.; KIRNER, C. FLARAS 1.0- Flash Augmented Reality Authoring System. 2012. Disponível no website: http//www.ckirner.com/faras Acesso em 29/junho/2012.

\footnotetext{
${ }^{5}$ Imagem do Armazem 3D do Hospital das Clinicas da Unicamp, disponível no website: http://sketchup.google.com/3dwarehouse/details?mid=c9b8e0ca6bedf69af984938119ddcc8c\&prevstart=0 acesso em 30/maio/2013. Esse prédio não consta na rota acessível, porém foi utilizada apenas para ilustrar e enriquecer o SI3D-DV. O som e a LIBRAS foram suprimidas, por motivo técnico.
} 
II Congresso Brasileiro de Informática na Educação (CBIE 2013)

Workshops (WCBIE 2013)

WATAYA, R.S. et. al. Usando Realidade Aumentada em um Sistema de Percepção 3D para Deficientes Visuais. Workshop de Realidade Virtual e Aumentada - $6^{\circ}$ WRVA, UniSanta, 2010, Santos/SP. 